\title{
Linear Mean Estimates for the 3D Non-Autonomous Brinkman-Forchheimer-Extended-Darcy Equations with Singularly Oscillating Forces
}

\author{
Xueying Chen, Chaosheng Zhu* \\ School of Mathematics and Statistics, Southwest University, Chongqing, China \\ Email: ${ }^{\star}$ zcs@swu.edu.cn
}

How to cite this paper: Chen, X.Y. and Zhu, C.S. (2022) Linear Mean Estimates for the 3D Non-Autonomous BrinkmanForchheimer-Extended-Darcy Equations with Singularly Oscillating Forces. Open Access Library Journal, 9: e8347.

https://doi.org/10.4236/oalib.1108347

Received: January 3, 2022

Accepted: February 25, 2022

Published: February 28, 2022

Copyright $\odot 2022$ by author(s) and Open Access Library Inc.

This work is licensed under the Creative Commons Attribution International License (CC BY 4.0).

http://creativecommons.org/licenses/by/4.0/

\begin{abstract}
In this paper, we prove the existence of global strong solutions for the three-dimensional nonautonomous Brinkman-Forchheimer-extended-Darcy equation with singularly oscillating and show that the strong solutions are unique. In addition, we also give general estimates for its auxiliary linear equation; finally, we derive the oscillatory averaged estimates of the equation from the results of these general estimates.
\end{abstract}

\section{Subject Areas \\ Partial Differential Equation}

\section{Keywords}

Singularly Oscillating Forces, Brinkman-Forchheimer-Extended-Darcy

Equations, Existence and Uniqueness of Solutions,

Oscillatory Averaged Estimation

\section{Introduction}

Let $\rho \in[0,1)$ be a fixed parameter, $\Omega \subset \mathbb{R}^{3}$ is a bounded domain, and the boundary $\partial \Omega$ is smooth. We study the $3 \mathrm{D}$ Non-autonomous Linearization Brinkman-Forchheimer-extended-Darcy Equations with singularly oscillating forces in $\Omega$ [1] [2] [3]:

$$
\left\{\begin{array}{l}
\partial_{t} u-\mu \Delta u+(u \cdot \nabla) u+a u+b|u|^{2 \alpha} u+c|u|^{2 \beta} u+\nabla p=f_{0}(t, x)+\varepsilon^{-\rho} f_{1}(t / \varepsilon, x), \\
\nabla \cdot u=0, \quad x \in \Omega, \\
\left.u(x, t)\right|_{t=0}=0, \\
\left.u(x, t)\right|_{\partial \Omega}=0,
\end{array}\right.
$$


where $a>0, b>0, c>0, \alpha, \beta \in[0, \infty), \quad \mu>0$ is the kinematic viscosity coefficient of the fluid, unknown function $u=u(x, t)=\left(u_{1}(x, t), u_{2}(x, t), u_{3}(x, t)\right)$ represents the velocity vector field of the fluid, $p=p(x, t)$ indicates pressure. Note that when $b, c=0$, Equation (1.1) is a Navier-Stokes equation with singular oscillatory force.

Combined with Equation (1.1), we study the following averaged Brinkman-Forchheimer-extended-Darcy equation (corresponding to the limit case $\varepsilon=0)$ :

$$
\left\{\begin{array}{l}
\partial_{t} u-\mu \Delta u+(u \cdot \nabla) u+a u+b|u|^{2 \alpha} u+c|u|^{2 \beta} u+\nabla p=f_{0}(t, x) \\
\nabla \cdot u=0, \quad x \in \Omega, \\
\left.u(x, t)\right|_{t=0}=0 \\
\left.u(x, t)\right|_{\partial \Omega}=0 .
\end{array}\right.
$$

Recording function

$$
f^{\varepsilon}(t, x) \equiv \begin{cases}f_{0}(t, x)+\varepsilon^{-\rho} f_{1}(t / \varepsilon, x), & 0<\varepsilon<1, \\ f_{0}(t, x), & \varepsilon=0 .\end{cases}
$$

Function $f_{0}(x, s), \quad f_{1}(x, s) \in L_{b}^{2}(\mathbb{R}, H)$, and $L_{b}^{2}(\mathbb{R}, H)$ is the translation bounded function in $L_{l o c}^{2}(\mathbb{R}, H)$, that is, there are two constants $M_{0}, M_{1} \geq 0$, making the following formula true:

$$
\begin{aligned}
\left\|f_{0}\right\|_{L_{b}^{2}}^{2} & \equiv \sup _{t \in R} \int_{t}^{t+1}\left\|f_{0}(s)\right\|^{2} \mathrm{~d} s=M_{0}^{2}, \\
\left\|f_{1}\right\|_{L_{b}^{2}}^{2} & \equiv \sup _{t \in R} \int_{t}^{t+1}\left\|f_{1}(s)\right\|^{2} \mathrm{~d} s=M_{1}^{2} .
\end{aligned}
$$

Define that

$$
Q^{\varepsilon} \equiv \begin{cases}M_{0}+2 M_{1} \varepsilon^{-\rho}, & 0<\varepsilon<1 \\ M_{0}, & \varepsilon=0 .\end{cases}
$$

Then, $\left\|f^{\varepsilon}\right\|_{L_{b}^{2}} \leq Q^{\varepsilon}$ can be obtained directly from (1.3). Note that when $\varepsilon \rightarrow 0$, the order of magnitude of $Q^{\varepsilon}$ is $\varepsilon^{-\rho}$.

Let's introduce the following function space [4] [5]

$$
\mathcal{V}=\left\{u \in\left(C_{0}^{\infty}(\Omega)\right)^{3}: \nabla u=0\right\}, \quad H=c l_{\left(L^{2}(\Omega)\right)^{3}} \mathcal{V}, \quad V=c l_{\left(H_{0}^{1}(\Omega)\right)^{3}} \mathcal{V},
$$

Here $c l_{X}$ represents the closure in space $X$, obviously, $H, V$ are separable Hilbert spaces, so that $H^{\prime}$ is the dual space of $H, V^{\prime}$ is the dual space of $V$, then $V \hookrightarrow H=H^{\prime} \hookrightarrow V^{\prime}$, the embedding is continuous and dense, and $\langle\cdot, \cdot\rangle$ represents the dual set between $V$ and $V^{\prime}$. For $H, V$ has the following inner product and norm respectively:

$$
\begin{aligned}
& (u, v)=\sum_{j=1}^{3} \int_{\Omega} u_{j}(x) v_{j}(x) \mathrm{d} x, \quad|\cdot|_{2}=(\cdot, \cdot)^{\frac{1}{2}}, \quad \forall u, v \in H, \\
& ((u, v))=\sum_{j=1}^{3} \int_{\Omega} \frac{\partial u_{j}}{\partial x_{i}} \frac{\partial v_{j}}{\partial x_{i}} \mathrm{~d} x, \quad\|\cdot\|=((\cdot, \cdot))^{\frac{1}{2}}, \quad \forall u, v \in V .
\end{aligned}
$$

Denoted by $|\cdot|_{p}$ represents the norm in $\mathbf{L}^{p}(\Omega),|\cdot|_{X}$ represents the norm in 
Banach space $X$. For $L^{p}(0, T ; x), 1 \leq p \leq \infty$, define the set of functions $f(t)$ on $(0, T)$ in $X$ so that $\int_{0}^{T}|f(t)|_{X}^{p} \mathrm{~d} t<\infty$ holds. The letter $C$ indicates a positive constant independent of the initial time.

The purpose of this paper is to prove the existence and uniqueness of the solution of the three-dimensional non-autonomous linearized Brinkman-Forchheimer-extended-Darcy equation with singular oscillatory force, and derive some estimates, and obtain the convergence of the corresponding equation compared with the average equation.

\section{Main Results and Proof}

The first and second equations of (1.1) can be written in the following form:

$$
\left\{\begin{array}{l}
\partial_{t} u+\mu A u+a u+B(u)+F(u)=f^{\varepsilon}(x, t), \\
\nabla \cdot u=0 .
\end{array}\right.
$$

where $A=-P \Delta$ is the Stokes operator and $P$ is the Leray orthogonal projection from $\mathbf{L}^{2}(\Omega)$ to $H ; \quad F(u)=P\left(b|u|^{2 \alpha} u+c|u|^{2 \beta} u\right)$. Define $\langle A u, v\rangle=((u, v))$; $B: V \times V \rightarrow V^{\prime}$ as a bilinear operator, $\langle B(u, v) w\rangle=b(u, v, w)$,

$$
B(u)=b(u, u), \quad b(u, v, w)=\sum_{i, j=1}^{3} \int_{\Omega} u_{i} \frac{\partial v_{j}}{\partial x_{i}} w_{j} \mathrm{~d} x .
$$

The following proves the existence and uniqueness of global solutions of Equation (2.1).

Theorem 2.1. Suppose $f^{\varepsilon} \in L_{\text {loc }}^{2}(\mathbb{R}, H)$ translational compactness, $\int_{0}^{T}\left|f^{\varepsilon}(t)\right|_{2}^{2} \mathrm{~d} t \leq C<+\infty$, any given $T>0$, there is a unique strong solution u for the initial boundary value problem of Equation (2.1),

$$
u \in C([0,+\infty) ; H) \cap L^{2}(0, T ; V) \cap L^{2 \alpha+2}\left(0, T ; L^{2 \alpha+2}(\Omega)\right) \cap L^{2 \beta+2}\left(0, T ; L^{2 \beta+2}(\Omega)\right)
$$

and

$$
\sup _{0 \leq t \leq T}|u(t)|_{2}^{2}+2 \mu \int_{0}^{T}|\nabla u|_{2}^{2} \mathrm{~d} t^{2}+2 b \int_{0}^{T}|u|_{2 \alpha+2}^{2 \alpha+2} \mathrm{~d} t+2 c \int_{0}^{T}|u|_{2 \beta+2}^{2 \beta+2} \mathrm{~d} t \leq \frac{2}{a} \int_{0}^{T}\left|f^{\varepsilon}(t)\right|_{2}^{2} \mathrm{~d} t .
$$

Proof. We use Galerkin approximation to prove this theorem. Due to the space $V$ is separable and space $C^{\infty}$ is dense in space $V$, there is a sequence $\omega_{1}, \omega_{2}, \cdots, \omega_{m}$ composed of elements in space $C^{\infty}$ which is free and completely belongs to space $V$. For any $m$, we define the following approximate solution:

$$
u_{m}=\sum_{i=1}^{m} g_{i m}(t) \omega_{i}(x)
$$

make the following formula true

$$
\begin{aligned}
& \left(u_{m}^{\prime}(t), \omega_{j}\right)+\mu\left(\nabla u_{m}(t), \omega_{j}\right)+\left(u_{m}(t) \cdot \nabla u_{m}(t), \omega_{j}\right)+\left(a u_{m}(t), \omega_{j}\right) \\
& +\left(b\left|u_{m}\right|^{2 \alpha}(t), \omega_{j}\right)+\left(b\left|u_{m}\right|^{2 \beta}(t), \omega_{j}\right)=\left(f^{\varepsilon}(t), \omega_{j}\right),
\end{aligned}
$$

where $t \in[0, T], \quad j=1,2, \cdots, m$, and in space $L^{2}, u_{0 m} \rightarrow 0$ as $m \rightarrow \infty$. Multiply both sides of the above equation by $g_{i m}(t)$ at the same time and sum $j=1, \cdots, m$ : 


$$
\begin{aligned}
& \frac{1}{2} \frac{\mathrm{d}}{\mathrm{d} t}\left|u_{m}\right|_{2}^{2}+\mu\left|\nabla u_{m}\right|_{2}^{2}+a\left|u_{m}\right|_{2}^{2}+b\left|u_{m}\right|_{2 \alpha+2}^{2 \alpha+2}+c\left|u_{m}\right|_{2 \beta+2}^{2 \beta+2} \\
& =\left(f^{\varepsilon}(t), u_{m}\right) \leq \frac{1}{a}\left|f^{\varepsilon}(t)\right|_{2}^{2}+a\left|u_{m}\right|_{2}^{2},
\end{aligned}
$$

namely

$$
\frac{1}{2} \frac{\mathrm{d}}{\mathrm{d} t}\left|u_{m}\right|_{2}^{2}+\mu\left|\nabla u_{m}\right|_{2}^{2}+b\left|u_{m}\right|_{2 \alpha+2}^{2 \alpha+2}+c\left|u_{m}\right|_{2 \beta+2}^{2 \beta+2} \leq \frac{1}{a}\left|f^{\varepsilon}(t)\right|_{2}^{2},
$$

for any $u, v \in V$, there are $((u \cdot \nabla) v, v)=0$. The above equation can be obtained by integrating on $[0, T]$ :

$\sup _{0 \leq t \leq T}|u(t)|_{2}^{2}+2 \mu \int_{0}^{T}|\nabla u|_{2}^{2} \mathrm{~d} t^{2}+2 b \int_{0}^{T}|u|_{2 \alpha+2}^{2 \alpha+2} \mathrm{~d} t+2 c \int_{0}^{T}|u|_{2 \beta+2}^{2 \beta+2} \mathrm{~d} t \leq \frac{2}{a} \int_{0}^{T}\left|f^{\varepsilon}(t)\right|_{2}^{2} \mathrm{~d} t$.

From the above formula, it is easy to obtain the existence by using the proof method similar to the Navier-Stokes equation with damping [6].

Now prove a priori estimate. Multiply both ends of Equation (2.1) by $u_{t},-\Delta u$, and then integrate on $\Omega$ :

$$
\begin{aligned}
& \frac{\mu}{2} \frac{\mathrm{d}}{\mathrm{d} t} \int_{\Omega}|\nabla u|^{2} \mathrm{~d} x+\int_{\Omega}\left|u_{t}\right|^{2} \mathrm{~d} x+\frac{a}{2} \frac{\mathrm{d}}{\mathrm{d} t} \int_{\Omega}|u|^{2} \mathrm{~d} x+\frac{b}{2 \alpha+2} \frac{\mathrm{d}}{\mathrm{d} t} \int_{\Omega}|u|^{2 \alpha+2} \mathrm{~d} x \\
& +\frac{c}{2 \beta+2} \frac{\mathrm{d}}{\mathrm{d} t} \int_{\Omega}|u|^{2 \beta+2} \mathrm{~d} x=\left(f^{\varepsilon}, u_{t}\right), \\
& \frac{1}{2} \frac{\mathrm{d}}{\mathrm{d} t} \int_{\Omega}|\nabla u|^{2} \mathrm{~d} x+\mu \int_{\Omega}|\Delta u|^{2} \mathrm{~d} x+a \int_{\Omega}|\nabla u|^{2} \mathrm{~d} x+b \int_{\Omega}|u|^{2 \alpha}|\nabla u|^{2} \mathrm{~d} x \\
& +\left.\left.\frac{4 b \alpha}{(2 \alpha+2)^{2}} \int_{\Omega}|\nabla| u\right|^{\frac{2 \alpha+2}{2}}\right|^{2} \mathrm{~d} x+c \int_{\Omega}|u|^{2 \beta}|\nabla u|^{2} \mathrm{~d} x+\left.\left.\frac{4 c \beta}{(2 \beta+2)^{2}} \int_{\Omega}|\nabla| u\right|^{\frac{\beta+2}{2}}\right|^{2} \mathrm{~d} x \\
& =-\int_{\Omega} f^{\varepsilon} \cdot \Delta u \mathrm{~d} x,
\end{aligned}
$$

adding the two formulas and applying Hölder inequality and Young inequality, we can deduce

$$
\begin{aligned}
& \frac{\mu+1}{2} \frac{\mathrm{d}}{\mathrm{d} t} \int_{\Omega}|\nabla u|^{2} \mathrm{~d} x+\int_{\Omega}\left|u_{t}\right|^{2} \mathrm{~d} x+\frac{a}{2} \frac{\mathrm{d}}{\mathrm{d} t} \int_{\Omega}|u|^{2} \mathrm{~d} x+\frac{b}{2 \alpha+2} \frac{\mathrm{d}}{\mathrm{d} t} \int_{\Omega}|u|^{2 \alpha+2} \mathrm{~d} x \\
& +\frac{c}{2 \beta+2} \frac{\mathrm{d}}{\mathrm{d} t} \int_{\Omega}|u|^{2 \beta+2} \mathrm{~d} x+\mu \int_{\Omega}|\Delta u|^{2} \mathrm{~d} x+a \int_{\Omega}|\nabla u|^{2} \mathrm{~d} x+b \int_{\Omega}|u|^{2 \alpha}|\nabla u|^{2} \mathrm{~d} x \\
& +\left.\left.\frac{4 b \alpha}{(2 \alpha+2)^{2}} \int_{\Omega}|\nabla| u\right|^{2 \alpha+2}\right|^{2} \mathrm{~d} x+c \int_{\Omega}|u|^{2 \beta}|\nabla u|^{2} \mathrm{~d} x+\left.\left.\frac{4 c \beta}{(2 \beta+2)^{2}} \int_{\Omega}|\nabla| u\right|^{2 \beta+2}\right|^{2} \mathrm{~d} x \\
& \leq \frac{1}{2}\left|u_{t}\right|_{2}^{2}+\frac{\mu}{2}|\Delta u|_{2}^{2}+\frac{1}{2}\left|f^{\varepsilon}\right|_{2}^{2}+\frac{1}{2 \mu}\left|f^{\varepsilon}\right|_{2}^{2},
\end{aligned}
$$

therefore

$$
\begin{aligned}
& \frac{\mu+1}{2} \frac{\mathrm{d}}{\mathrm{d} t} \int_{\Omega}|\nabla u|^{2} \mathrm{~d} x+\frac{1}{2} \int_{\Omega}|u|^{2} \mathrm{~d} x+\frac{a}{2} \frac{\mathrm{d}}{\mathrm{d} t} \int_{\Omega}|u|^{2} \mathrm{~d} x+\frac{b}{2 \alpha+2} \frac{\mathrm{d}}{\mathrm{d} t} \int_{\Omega}|u|^{2 \alpha+2} \mathrm{~d} x \\
& +\frac{c}{2 \beta+2} \frac{\mathrm{d}}{\mathrm{d} t} \int_{\Omega}|u|^{2 \beta+2} \mathrm{~d} x+\frac{\mu}{2} \int_{\Omega}|\Delta u|^{2} \mathrm{~d} x+a \int_{\Omega}|\nabla u|^{2} \mathrm{~d} x+b \int_{\Omega}|u|^{2 \alpha}|\nabla u|^{2} \mathrm{~d} x \\
& +\left.\left.\frac{4 b \alpha}{(2 \alpha+2)^{2}} \int_{\Omega}|\nabla| u\right|^{\alpha+1}\right|^{2} \mathrm{~d} x+c \int_{\Omega}|u|^{2 \beta}|\nabla u|^{2} \mathrm{~d} x+\left.\left.\frac{4 c \beta}{(2 \beta+2)^{2}} \int_{\Omega}|\nabla| u\right|^{\beta+1}\right|^{2} \mathrm{~d} x \\
& \leq \frac{1}{2}\left|f^{\varepsilon}\right|_{2}^{2}+\frac{1}{2 \mu}\left|f^{\varepsilon}\right|_{2}^{2},
\end{aligned}
$$


integrate on 0 to $T$

$$
\begin{aligned}
& (\mu+1) \sup _{0 \leq t \leq T}|\nabla u(t)|_{2}^{2}+a \sup _{0 \leq t \leq T}|u(t)|_{2}^{2}+\frac{2 b}{2 \alpha+2} \sup _{0 \leq t \leq T}|u(t)|_{2 \alpha+2}^{2 \alpha+2} \\
& +\frac{2 c}{2 \beta+2} \sup _{0 \leq t \leq T}|u(t)|_{2 \beta+2}^{2 \beta+2}+\int_{0}^{T} \int_{\Omega}\left|u_{t}\right|^{2} \mathrm{~d} x \mathrm{~d} t \\
& +\mu \int_{0}^{T} \int_{\Omega}|\Delta u|^{2} \mathrm{~d} x \mathrm{~d} t+2 a \int_{0}^{T} \int_{\Omega}|\nabla u|^{2} \mathrm{~d} x \mathrm{~d} t \\
& +\left.\left.2 b \int_{0}^{T} \int_{\Omega}|| \nabla u|| u\right|^{\alpha}\right|^{2} \mathrm{~d} x \mathrm{~d} t+\left.\left.\frac{8 b \alpha}{(2 \alpha+2)^{2}} \int_{0}^{T} \int_{\Omega}|| \nabla u\right|^{\alpha+1}\right|^{2} \mathrm{~d} x \mathrm{~d} t \\
& +\left.\left.2 c \int_{0}^{T} \int_{\Omega}|| \nabla u|| u\right|^{\beta}\right|^{2} \mathrm{~d} x \mathrm{~d} t+\left.\left.\frac{8 c \beta}{(2 \beta+2)^{2}} \int_{0}^{T} \int_{\Omega}|| \nabla u\right|^{\beta+1}\right|^{2} \mathrm{~d} x \mathrm{~d} t \\
& \leq C \int_{0}^{T}|f|_{2}^{2} \mathrm{~d} t \leq C .
\end{aligned}
$$

The uniqueness is proved below. Assuming that under the same initial conditions, because of Divergence free, Equation (2.1) has two strong solutions $(u, p),(\bar{u}, \bar{p})$ satisfies

$$
\begin{aligned}
& \left(u_{t}, \Phi\right)+\mu \int_{\Omega} \nabla u: \nabla \Phi \mathrm{d} x-\int_{\Omega}(u \cdot \nabla) u \Phi \mathrm{d} x+a(u, \Phi) \\
& +b \int_{\Omega}|u|^{2 \alpha} u \Phi \mathrm{d} x+c \int_{\Omega}|u|^{2 \beta} u \Phi \mathrm{d} x=f^{\varepsilon}(x, t), \\
& \left(\bar{u}_{t}, \Phi\right)+\mu \int_{\Omega} \nabla \bar{u}: \nabla \Phi \mathrm{d} x-\int_{\Omega}(\bar{u} \cdot \nabla) \bar{u} \Phi \mathrm{d} x+a(\bar{u}, \Phi) \\
& +b \int_{\Omega}|\bar{u}|^{2 \alpha} \bar{u} \Phi \mathrm{d} x+c \int_{\Omega}|\bar{u}|^{2 \beta} \bar{u} \Phi \mathrm{d} x=f^{\varepsilon}(x, t),
\end{aligned}
$$

where $\Phi \in C^{\infty}\left([0, T] \times R^{3}\right), \quad A: B=\sum_{i, j=1}^{3} a_{i j} b_{i j}$. Subtracting (2.4) from (2.3) and letting $\Phi=u-\bar{u}$, it can be obtained

$$
\begin{aligned}
& \frac{1}{2} \frac{\mathrm{d}}{\mathrm{d} t}|u-\bar{u}|_{2}^{2}+\mu|\nabla(u-\bar{u})|_{2}^{2}+a|u-\bar{u}|_{2}^{2}+\left.b|| u\right|^{\alpha}|u-\bar{u}|_{2}^{2}+\left.c|| u\right|^{\beta}|u-\bar{u}|_{2}^{2} \\
& \leq \int_{\Omega}|u-\bar{u}|_{2}^{2}|\nabla \bar{u}| \mathrm{d} x+\left.b \int_{\Omega}|u-\bar{u}||\bar{u}||| u\right|^{2 \alpha}-|\bar{u}|^{2 \alpha} \mid \mathrm{d} x \\
& \quad+\left.c \int_{\Omega}|u-\bar{u}||\bar{u}||| u\right|^{2 \beta}-|\bar{u}|^{2 \beta} \mid \mathrm{d} x \\
& \equiv I_{1}+I_{2}+I_{3},
\end{aligned}
$$

where we use $((u \cdot \nabla) u, v)=0, u \in V, v \in V$. Then we use Hölder inequality and Sobolev inequality

$$
\begin{aligned}
I_{1} & \leq|u-\bar{u}|_{4}^{2}|\nabla \bar{u}|_{2} \\
& \leq C\left(|\nabla(u-\bar{u})|_{2}^{\frac{3}{4}}|u-\bar{u}|_{2}^{\frac{1}{4}}\right)^{2}|\nabla \bar{u}|_{2} \\
& \leq C|\nabla(u-\bar{u})|_{2}^{\frac{3}{2}}|u-\bar{u}|_{2}^{\frac{1}{2}}|\nabla \bar{u}|_{2} \\
& \leq \varepsilon|\nabla(u-\bar{u})|_{2}^{2}+C|u-\bar{u}|_{2}^{2}|\nabla \bar{u}|_{2}^{4},
\end{aligned}
$$




$$
\begin{aligned}
I_{2} & \leq\left. b \int_{\Omega}|u-\bar{u}||\bar{u}||| u\right|^{2 \alpha}-|\bar{u}|^{2 \alpha} \mid \mathrm{d} x \\
& \leq\left. C(2 \alpha) \int_{\Omega}|u-\bar{u}||| u\right|^{2 \alpha-1}+|\bar{u}|^{2 \alpha-1}|| u-\bar{u}|| \bar{u} \mid \mathrm{d} x \\
& \leq\left. C|u-\bar{u}|_{4}^{2}|\bar{u}|_{6}|| u\right|^{2 \alpha-1}+\left.|\bar{u}|^{2 \alpha-1}\right|_{3} \\
& \leq\left. C\left(|\nabla(u-\bar{u})|_{2}^{\frac{3}{4}}|u-\bar{u}|_{2}^{\frac{1}{4}}\right)^{2}|\bar{u}|_{6}|| u|+| \bar{u}\right|_{3(2 \alpha-1)} ^{2 \alpha-1} \\
& \leq C|\nabla(u-\bar{u})|_{2}^{\frac{3}{2}}|u-\bar{u}|_{2}^{\frac{1}{2}}|\bar{u}|_{6}|| u|+| \bar{u} \|_{3(2 \alpha-1)}^{2 \alpha-1} \\
& \leq \varepsilon|\nabla(u-\bar{u})|_{2}^{2}+C|u-\bar{u}|_{2}^{2}|\bar{u}|_{6}^{4}|| u|+| \bar{u} \|_{3(2 \alpha-1)}^{4(2 \alpha-1)}
\end{aligned}
$$

Similarly, we can get the estimate of $I_{3}$. Substitute the estimate $I_{1}, I_{2}, I_{3}$ into the inequality (2.4), select $\varepsilon=\frac{\mu}{6}$, it can be obtained

$$
\begin{aligned}
& \frac{\mathrm{d}}{\mathrm{d} t}|u-\bar{u}|_{2}^{2}+\mu|\nabla(u-\bar{u})|_{2}^{2}+2 a|u-\bar{u}|_{2}^{2}+\left.2 b|| u\right|^{\alpha}|u-\bar{u}|_{2}^{2}+\left.2 c|| u\right|^{\beta}|u-\bar{u}|_{2}^{2} \\
& \leq C|u-\bar{u}|_{2}^{2}\left(|\nabla \bar{u}|_{2}^{4}+|\bar{u}|_{6}^{4}\left[|u|_{3(2 \alpha-1)}^{4(2 \alpha-1)}+|\bar{u}|_{3(2 \alpha-1)}^{4(2 \alpha-1)}+|u|_{3(2 \beta-1)}^{4(2 \beta-1)}+|\bar{u}|_{3(2 \beta-1)}^{4(2 \beta-1)}\right]\right) .
\end{aligned}
$$

Note that from the Gagliardo-Nirenberg inequality and a priori estimation, we can derive:

$$
\begin{aligned}
\int_{0}^{T}\|u\|_{3(2 \alpha-1)}^{4(2 \alpha-1)} \mathrm{d} t & \leq \int_{0}^{T}|u|_{2 \alpha+2}^{\frac{4(2 \alpha+1)(\alpha+1)}{\alpha+4}}|\Delta u|_{2}^{\frac{4(4 \alpha-5)}{\alpha+4}} \mathrm{~d} t \\
& \leq C \sup _{0 \leq t \leq T}|u|_{2 \alpha+2}^{\frac{4(2 \alpha+1)(\alpha+1)}{\alpha+4}}\left(\int_{0}^{T}|\Delta u|_{2}^{2} \mathrm{~d} t\right)^{\frac{2(4 \alpha-5)}{\alpha+4}} T^{\frac{28-14 \alpha}{\alpha+4}} \leq C,
\end{aligned}
$$

using $\bar{u}$ instead of $u$ in (2.7) also applies to the above estimation. In Equation (2.6), we have a limit on $\alpha$ making $0 \leq \frac{4(4 \alpha-5)}{\alpha+4} \leq 2$ establish, namely $\frac{5}{4} \leq \alpha \leq 2$, similarly, we limit $\frac{5}{4} \leq \beta \leq 2$. Substitute (2.7) into (2.6) and apply Gronwall inequality, it can be obtained that under the restriction of the above formula, for almost everywhere $(x, t) \in \Omega \times[0, T]$, there are $u=\bar{u}$. Theorem 2.1 proved.

Next we consider the auxiliary linear equation of Equation (2.1):

$$
Y_{t}+\mu A Y+a Y=K(t),\left.\quad Y\right|_{t=0}=0,
$$

we get the following theorem.

Theorem 2.2. Suppose $K \in L_{\text {loc }}^{2}(\mathbb{R}, H)$, then the problem (2.8) has a unique strong solution:

$$
Y \in C([0, T] ; V) \cap L^{2}(0, T ; V), \quad Y_{t} \in C\left([0, T] ; V^{\prime}\right) .
$$

and for $\forall t \geq 0$, the following inequality is satisfied:

$$
\begin{gathered}
\|Y(t)\|^{2} \leq C \int_{0}^{t} \mathrm{e}^{-C \mu(t-s)}|K(s)|_{2}^{2} \mathrm{~d} s \\
\int_{t}^{t+1}|Y(s)|_{2}^{2} \mathrm{~d} s \leq C\left(|Y(t)|_{2}^{2}+\int_{t}^{t+1}|K(s)|_{2}^{2} \mathrm{~d} s\right) .
\end{gathered}
$$


Proof. First, the inner product of Equation (2.8) and $Y$ can be obtained:

$$
\begin{aligned}
\frac{1}{2} \frac{\mathrm{d}}{\mathrm{d} t}|Y|_{2}^{2}+\mu \lambda_{1}|Y|_{2}^{2}+a|Y|_{2}^{2} & =\frac{1}{2} \frac{\mathrm{d}}{\mathrm{d} t}|Y|_{2}^{2}+\mu\|Y\|^{2}+a|Y|_{2}^{2} \\
& =(K(t), Y) \leq \frac{1}{a}|K|_{2}^{2}+a|Y|_{2}^{2},
\end{aligned}
$$

namely

$$
\frac{\mathrm{d}}{\mathrm{d} t}|Y|_{2}^{2}+2 \mu \lambda_{1}|Y|_{2}^{2} \leq \frac{2}{a}|K|_{2}^{2} .
$$

integrate the above formula on $[t, t+1]$, and then use Poincaré inequality:

$$
\begin{aligned}
2 \mu \lambda_{1} \int_{t}^{t+1}|Y(s)|^{2} \mathrm{~d} s & \leq-|Y(t+1)|_{2}^{2}+|Y(t)|_{2}^{2}+\frac{2}{a} \int_{t}^{t+1}|K(s)|_{2}^{2} \mathrm{~d} s \\
& \leq|Y(t)|_{2}^{2}+\frac{2}{a} \int_{t}^{t+1}|K(s)|_{2}^{2} \mathrm{~d} s,
\end{aligned}
$$

namely

$$
\int_{t}^{t+1}|Y(s)|_{2}^{2} \mathrm{~d} s \leq C\left(|Y(t)|_{2}^{2}+\int_{t}^{t+1}|K(s)|_{2}^{2} \mathrm{~d} s\right) .
$$

take Equation (2.8) and $A Y$ as inner product to obtain:

$$
\frac{1}{2} \frac{\mathrm{d}}{\mathrm{d} t}\|Y\|^{2}+\mu|A Y|_{2}^{2}+a\|Y\|^{2}=(K(t), A Y) \leq \frac{1}{\mu}|K|_{2}^{2}+\mu|A Y|_{2}^{2},
$$

thus, it can be obtained:

$$
\frac{1}{2} \frac{\mathrm{d}}{\mathrm{d} t}\|Y\|^{2}=-a\|Y\|^{2}+\frac{1}{\mu}|K|_{2}^{2} .
$$

apply Gronwall inequality to the above formula in the interval $[0, t]$ :

$$
\|Y(t)\|^{2} \leq C \int_{0}^{t} \mathrm{e}^{-C \mu(t-s)}|K(s)|_{2}^{2} \mathrm{~d} s .
$$

therefore, the existence of solutions can be deduced by Galerkin approximation method. Theorem 2.2 is proved.

Let $K(t, 0)=\int_{0}^{t} k(s) \mathrm{d} s, t \geq 0$, next, we prove that the solution of the linear equation of singular oscillatory force converges to the solution of the average equation.

Theorem 2.3. Let $k \in L_{\text {loc }}^{2}(\mathbb{R}, H)$, suppose there is a constant $l \geq 0$ satisfying

$$
\sup _{t \geq 0}\left\{|K(t, 0)|_{2}^{2}+\int_{t}^{t+1}|K(s, 0)|_{2}^{2} \mathrm{~d} s\right\} \leq l^{2},
$$

then the solution $Y(t)$ of the linear equation with singular oscillation force

$$
Y_{t}+\mu A X+a Y=k(t / \varepsilon),\left.\quad Y\right|_{t=0}=0
$$

with $\varepsilon \in(0,1)$, for $\forall t \geq 0$, satisfy the following inequality

$$
\|Y(t)\|^{2}+\int_{t}^{t+1}|Y(s)|_{2}^{2} \mathrm{~d} s \leq C l^{2} \varepsilon^{2},
$$

where $C>0$ and not related to $K$.

Proof. The proof of this theorem is similar to literature [7], for the conveni- 
ence of readers, a summary of the proof is given here. Firstly

$$
K_{\varepsilon}(t)=\int_{0}^{t} k(s / \varepsilon) \mathrm{d} s=\varepsilon \int_{0 / \varepsilon}^{t / \varepsilon} k(s) \mathrm{d} s=\varepsilon K(t / \varepsilon, 0 / \varepsilon),
$$

then the following estimate of $K_{\varepsilon}(t)$ can be derived from Equation (2.11): $\sup _{t \geq 0}\left|K_{\varepsilon}(t)\right|_{2} \leq l \varepsilon$ and

$$
\int_{t}^{t+1}\left|K_{\varepsilon}(s)\right|_{2}^{2} \mathrm{~d} s \leq C \varepsilon^{2} \sup _{t \geq 0} \int_{t}^{t+1}|K(s, 0)|_{2}^{2} \mathrm{~d} s \leq C l^{2} \varepsilon^{2},
$$

from Theorem 2.2, we can deduce that:

$$
\int_{0}^{t} \mathrm{e}^{-C \mu(t-s)}\left|K_{\varepsilon}(s)\right|_{2}^{2} \mathrm{~d} s \leq \frac{1}{1-\mathrm{e}^{-C \mu}} \sup _{t \geq 0} \int_{t}^{t+1}\left|K_{\varepsilon}(s)\right|_{2}^{2} \mathrm{~d} s \leq C l^{2} \varepsilon^{2} .
$$

therefore, by (2.9), (2.10), (2.13) and Poincaré inequality we can know:

$$
\|Y(t)\|^{2} \leq C l^{2} \varepsilon^{2}, \quad \int_{t}^{t+1}|Y(s)|_{2}^{2} \mathrm{~d} s \leq C\left(|Y(t)|_{2}^{2}+\int_{t}^{t+1}|K(s)|_{2}^{2} \mathrm{~d} s\right) \leq C l^{2} \varepsilon^{2} .
$$

Equation (2.12) integrates time from 0 to $t, \partial_{t} Y+\mu A Y+a Y=K_{\varepsilon}(t),\left.Y\right|_{t=0}=0$. It can be derived from (2.14):

$$
|Y(t)|_{2}^{2}+\|Y(t)\|^{2}+\int_{t}^{t+1}|Y(s)|_{2}^{2} \mathrm{~d} s \leq C l^{2} \varepsilon^{2} .
$$

Theorem 2.3 proved.

\section{Conflicts of Interest}

The authors declare no conflicts of interest.

\section{References}

[1] Titi, E.S. and Trabelsi, S. (2019) Global Well-Posedness of a 3D MHD Model in Porous Media. Geometric Mechanics, 11, 621-637. https://doi.org/10.3934/jgm.2019031

[2] Hsu, C. and Cheng, P. (1990) Thermal Dispersion in a Porous Medium. International Journal of Heat and Mass Transfer, 33, 1587-1597. https://doi.org/10.1016/0017-9310(90)90015-M

[3] Wei, J., Li, Y. and Zhuo, X. (2016) The Existence and Stability of Synchronization Solution of Non-Autonomous Equations with Multiple Delays. Journal of Applied Mathematics and Physics, 4, 1294-1299. https://doi.org/10.4236/jamp.2016.47136

[4] Nield, D. (1991) The Limitations of the Brinkman-Forchheimer Equation in Modeling Flow in a Saturated Porous Medium and at an Interface. International Journal of Heat and Fluid Flow, 12, 269-272. https://doi.org/10.1016/0142-727X(91)90062-Z

[5] Vafai, K. and Thiyagaraja, R. (1987) Analysis of Flow and Heat Transfer at the Interface Region of a Porous Medium. International Journal of Heat and Mass Transfer, 30, 1391-1405. https://doi.org/10.1016/0017-9310(87)90171-2

[6] Cai, X.J. and Jiu, Q.S. (2008) Weak and Strong Solutions for the Incompressible NavierCStokes Equations with Damping. Journal of Mathematical Analysis and Applications, 343, 799-809. https://doi.org/10.1016/j.jmaa.2008.01.041

[7] Wang, H.J. and Wang, X.S. (2014) Some Estimates for the 3D Non-Autonomous Linearization Brinkman-Forchheimer Equation with Singularly Oscillating. International Journal of Contemporary Mathematical Sciences, 9, 43-49. https://doi.org/10.12988/ijcms.2014.311121 